Dab der dynamische accent die ursache des stimmhaftwerdens von consonanten sein kann, läßt sich auch an den militiirischen commandos beobachten. Link-zum = 'links um', recht-zum = 'rechts um', ganze abteilung - gehort $=$ 'kehrt' hat wohl jeder einmal vernommen, der ein ohr dafür besitzt. - Daß der kasernenhof überhaupt ein ergiebiges feld für lautliche studien war, sei hier nur beiläufig erwähnt.

Es kann nun allerdings nicht ohne weiteres behauptet werden, daß der besprochene mundartliche und diese militärischen fälle den unter dem historischen Vernerschen gesetz begriffenen gleich sind, m. a. w. daß der dynamische accent auch für die letzteren bedingung gewesen wäre. Es ist eine mahnung zur vorsicht, wenn in tonsprachen eine ursächliche beziehung $z$ wischen stimmhaftigkeit oder stimmlosigkeit anlautender consonanten und tonhöhe besteht, wie $z$. b. im altchinesischen eine hohe gruppe mit stimmloser, eine tiefe gruppe mit stimmhafter consonanz im anlaut vorhanden war, wovon die heutigen dialekte noch spuren aufweisen. Da aber einmal noch nicht feststeht, ob das urindogermanische überhaupt eine reine tonsprache war oder nicht, da weiter für die zeit der wirkung des historischen Vernerschen gesetzes zum mindesten ein übergang des germanischen zum dynamischen accent angesetzt werden $m u ß$, und da drittens im historischen germanischen die immer stärker hervortretende wirkung des letzteren zu erkennen war und ist, so darf man dazu neigen, unsere fälle mit denen des historischen Vernerschen gesetzes gleichzusetzen.

HAMBURG.

KONRAD HENTRICH.

\title{
ZUM NOMINATIVUS UND ACCUSATIVUS SING. DER I $A$-STÄMME IM ALTENGLISCHEN.
}

Loewe meint Germ. sprachw.2 II, 11, daß urgermanisches -iaz bei den kurzsilbigen - $i a$-stämmen urenglisch $i$ ergeben habe und $\mathrm{daB} i$ zunächst consonantengemination hervorgerufen habe und dann geschwunden sei: das ist mir nicht einleuchtend. Ich möchte für den nom. und accus. sing. der ia-stämme 
folgende entwickelung annehmen. $i$ löste sich im auslaut in $i$ auf. Dafür stütze ich mich auf die parallele der ua-stämme; " ergab auslautend $u$ und blieb nach kurzer, consonantisch schließender stammsilbe im altenglischen als solches erhalten; jünger wurde $o$ daraus; vgl. z. b. meolu 'mehl' aus *melua-. Urgerm. *saz[u] iaz 'mann' aus idg. *soquiós sollte also urengl. ${ }^{*} s e z i$ ergeben, ebenso der accus. *saz[u]ian, *sezi. Nun wurde aber die consonantengemination bereits in urenglischer zeit aus dem genetiv und dativ, wo sie lantgesetzlich war, in dem nom. und accus. eingeführt, so daß $*$ scczi entstand. Jetzt stand $i$ aber hinter langer stammsilbe und mußte daher regelrecht schwinden. So kam secz heraus. Entsprechend beim nentrum im nom. und accus. statt des zu erwartenden *cuni, *cyni 'geschlecht' (aus urgerm. *kunian) nach dem gen. cynnces cynn aus *cynni. Von cz abgesehen wurde die gemination im auslaut vereinfacht; cynn statt $c y_{i}$ ist daher nochmalige analogiebildung nach cynnes, cynne. Für diese auffassung spricht auch das verhalten derjenigen $i a$-stämme, die ein $r$ vor $i$ haben; hier hat im gen. und dat. bekanntlich lautgesetzlich keine gemination stattgehabt; infolgedessen konnte hier auch der nom. und accus. nicht analogisch vom gen. und dat. beeinflußt werden. So liegt bei diesen stämmen die lautgesetzliche entwickelung ungetriibi, vor, wie $\mathrm{z}$. b. in heri, here 'heer' aus urgerm. *hariaz, *harian.

Altengl. cynn weicht von alts. ahd. kunni ab. Letztere stehen wohl unter dem einfluß des langsilbigen masculinums hirti und haben darnach $i$ beibehalten. Im altenglischen aber hat sich das neutrum $\operatorname{cyn}(n)$ an das masculinum $\sec _{\xi}$ angeschlossen. Dieses hat sich nicht nach ende (aus urgerm. *andiaz) gerichtet, sondern das $i$ lautgesetzlich schwinden lassen, wo\%u die langsilbigen $i$-stämme wie vyrm 'wurm' beigetragen haben.

MÜNCHEN.

F. KIECKERS. 